\title{
Correlation between Osteoporosis and Dental Caries in Korean Population
}

Ye-Soo Park ${ }^{1}$, Sang-Mo Hong 2 , Woon-Hwan Choi ${ }^{2}$, Byung-Moon Kang ${ }^{3}$, Ye-Yeon Won ${ }^{4}$

${ }^{l}$ Department of Orthopaedic Surgery, Guri Hospital, Hanyang University College of Medicine

${ }^{2}$ Department of Internal Medicine, Hanyang University College of Medicine

${ }^{3}$ Department of Obstetrics and Gynecology, Asan Medical Center, Ulsan University College of Medicine

${ }^{4}$ Department of Orthopaedic Surgery, Ajou University College of Medicine

\section{INTRODUCTION}

Aging is associated with a loss of systemic bone mass and an increased risk of especially the spine and hip. Therefore bone loss of teeth and ridge resorption can occur in the mouth. However, results of several studies investing association between systemic bone density and oral health have varied. In this study, we investigated the relationship between osteoporosis and dental caries with the Korea National Health and Nutrition Examination Survey (KNHANES) conducted in 2008-2010 in the Korean population.

\section{MATERIALS AND METHODS}

\section{Study design}

- The cross-sectional study

- Data acquired in the Korea National Health and Nutrition Examination Survey (KNHANES) (2008-2010).

2. Korea National Health and Nutrition Examination Survey

- A nationwide, population-based, and cross- sectionally designed health survey conducted by the Korea Centers for Disease Control and Prevention.

- A stratified, multistage, probability sampling design was used, with the selection made from sampling units based on geographical area, sex, and age-groups using household registries.

- Trained investigators conducted surveys in households and administered questionnaires to adults.

\section{Patient}

- Among KNHANES (2008-2009-2010) data, we analyzed $\underline{1803}$ women aged over 65 years old selected in all the 16 administrative districts of South Korea who had bone mineral density and dental examination data.

4. Bone mineral density

- By DXA methods with a QDR Discovery

(formerly the QDR 4500A) fan beam densitometer (Hologic, Inc., Bedford, MA)

- Among these DXA data, we used lumbar BMD (mean BMD of L1-L4).

\section{Dental examination}

- a dentist conducted a complete oral health examination

\section{RESULTS}

\begin{tabular}{|l|c|}
\hline \multicolumn{2}{|c|}{ Table1. Patient Characteristics } \\
\hline & Mean \pm SE or \% \\
\hline Number & 1803 \\
\hline Age $($ year $)$ & $72.39 \pm 0.17$ \\
\hline Height $(\mathrm{cm})$ & $150.9 \pm 0.2$ \\
\hline Weight $(\mathrm{kg})$ & $55.4 \pm 0.3$ \\
\hline BMI $\left(\mathrm{kg} / \mathrm{cm}^{2}\right)$ & $24.3 \pm 0.1$ \\
\hline FPG $(\mathrm{mg} / \mathrm{dl})$ & $104.6 \pm 0.9$ \\
\hline Diabetes mellitus (normal:prediabetes:diabetes) & $54.0: 24.1: 21.9$ \\
\hline Serum 25OHD $(\mathrm{ng} / \mathrm{mL})$ & $18.7 \pm 0.03$ \\
\hline Alcohol drinking $(\mathrm{over} 1$ time/week \%) & 5.5 \\
\hline Smoking $(\%)(\mathrm{no}:$ Ex-smoker:Current-smoker $)$ & $90.6: 5.5: 3.9$ \\
\hline Moderate Exercise $(\%)$ & 11.6 \\
\hline Lumbar BMD $(\mathrm{g} / \mathrm{cm} 2, \mathrm{~T}$ score $)$ & $0.749 \pm 0.004,-2.23 \pm 0.02$ \\
\hline Femur neck BMD $(\mathrm{g} / \mathrm{cm} 2, \mathrm{~T}$ score $)$ & $0.559 \pm 0.003,-2.28 \pm 0.03$ \\
\hline Dental caries & $26.7 \%$ \\
\hline *SE: standard error & \\
\hline
\end{tabular}

Table 2. Age, body weight, diabetes, cigarette smoking, and alcohol adjusted odd ratio of dental caries by bone mineral density

\begin{tabular}{|c|c|c|c|}
\hline \multirow{3}{*}{ Lumbar } & & Odd ratio & $95 \%$ confidence interval \\
\cline { 2 - 4 } & Normal & 1 (Ref.) & Ref. \\
\cline { 2 - 4 } & Osteopenia & 2.052 & $1.270-3.316$ \\
\cline { 2 - 4 } Femur neck & Osteoporosis & 1.928 & $1.182-3.143$ \\
\cline { 2 - 4 } & Normal & 1 (Ref.) & Ref. \\
\cline { 2 - 4 } & Osteopenia & 1.237 & $0.694-2.204$ \\
\cline { 2 - 4 } & Osteoporosis & 1.729 & $0.908-3.293$ \\
\hline
\end{tabular}

Figure 1. Prevalence of dental caries by bone mineral density

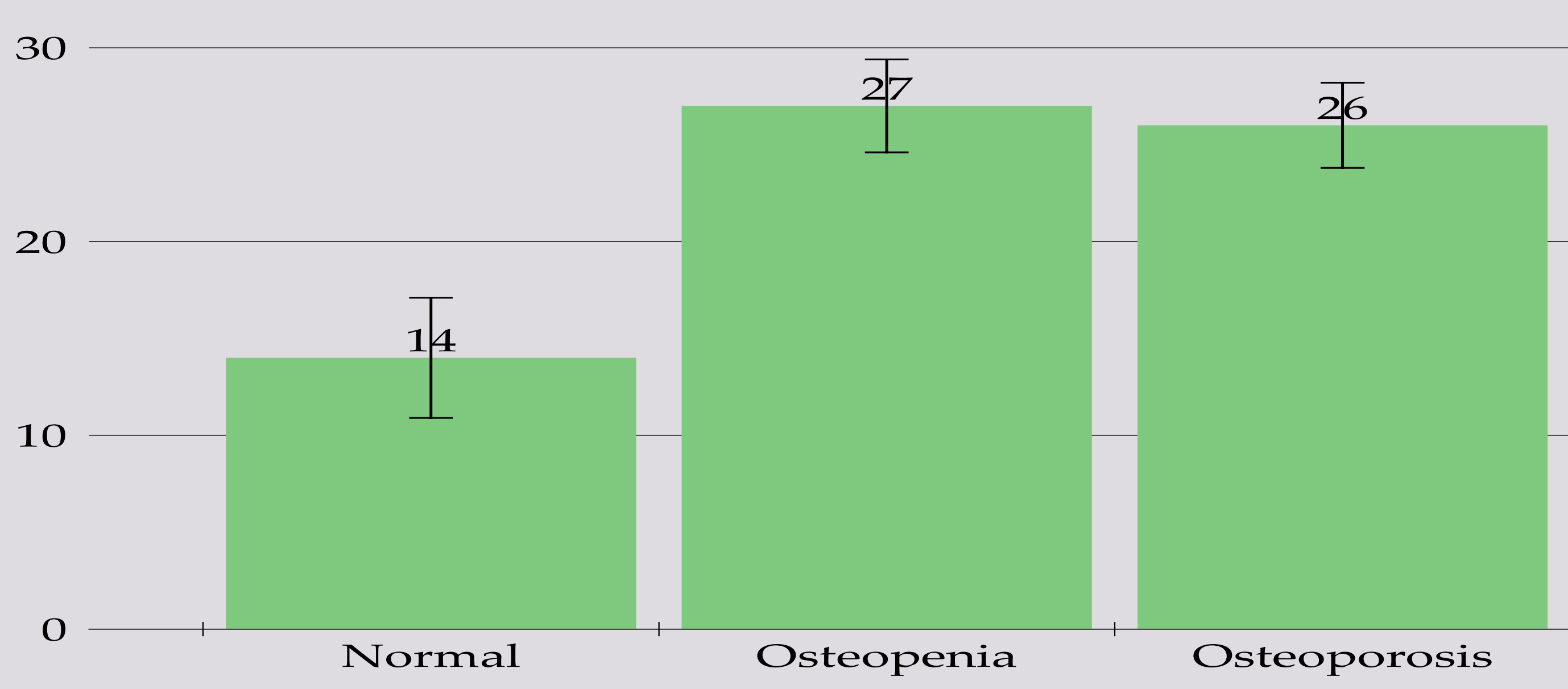

By Lumbar Bone mineral density

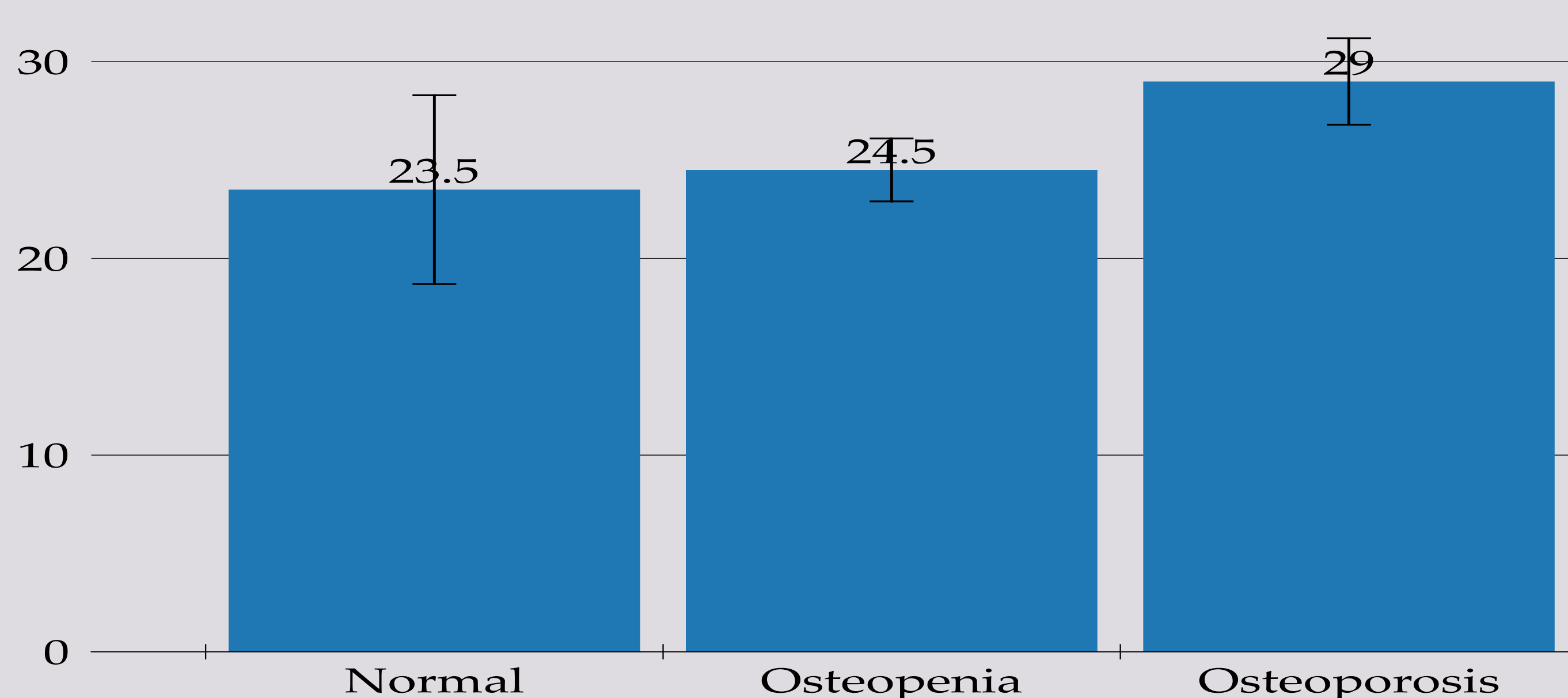

By Femur Neck Bone mineral density

\section{CONCLUSION}

Our results provide evidence of an association between osteoporosis and dental caries in aging women (>65 yrs.). The associations between bone density, and dental health require further research, particularly in longitudinal studies. 